\title{
Principal n-Ideals which Form Generalized Stone Nearlattices
}

\author{
Shiuly Akhter ${ }^{{ }^{*}}$ and A. S. A. Noor ${ }^{2}$ \\ ${ }^{1}$ Department of Mathematics, University of Rajshahi, Rajshahi-6205, Bangladesh \\ ${ }^{2}$ Department of Electronics and Communication Engineering, East-West University, 45 Mohakhali, \\ Dhaka-1212, Bangladesh
}

Received 1 June 2013, accepted in final revised form 9 January 2014

\begin{abstract}
In this paper, we give several characterizations of those $P_{n}(S)$ which are generalized Stone nearlattices in terms of n-ideals. We show that when $n$ is a central element of a nearlattice $S$ and $P_{n}(S)$ is a sectionally pseudocomplemented distributive nearlattice, then $P_{n}(S)$ is generalized Stone if and only if for any $x \in S,\langle x\rangle_{n}^{+} \vee\langle x\rangle_{n}^{++}=S$. Moreover, when $P_{n}(S)$ is sectionally pseudocomplemented distributive nearlattice, then we prove that $P_{n}(S)$ is generalized Stone if and only if each prime $n$-ideal contains a unique minimal prime $n$ ideal.

Keywords: Principal $n$-ideal; Minimal prime $n$-ideal; Central element; Generalized Stone nearlattice.

(C) 2014 JSR Publications. ISSN: 2070-0237 (Print); 2070-0245 (Online). All rights reserved.

doi: http://dx.doi.org/10.3329/jsr.v6i2.10818 J. Sci. Res. 6 (2), 233-241 (2014)
\end{abstract}

\section{Introduction}

Generalized Stone lattices have been studied by many authors including [1], [2], [3], [4] and [5]. On the other hand, minimal prime ideals and generalized Stone nearlattices have been studied by [6]. In this paper, we generalize several important results on generalized Stone nearlattices in terms of $n$-ideals.

A nearlattice $S$ is a meet semilattice with the property that any two elements possessing a common upper bound, have a supremum. Nearlattice $S$ is distributive if for all $x, y, z \in S, \quad x \wedge(y \vee z)=(x \wedge y) \vee(x \wedge z)$ provided $y \vee z$ exists. An element $n$ of a nearlattice $S$ is called medial if $m(x, n, y)=(x \wedge y) \vee(x \wedge n) \vee(y \wedge n)$ exists in $S$ for all $x, y \in S$. A nearlattice $S$ is called a medial nearlattice if $m(x, y, z)$ exists for all $x, y, z \in S$. An element $S$ of a nearlattice $S$ is called standard if for all $t, x, y \in S$,

$$
t \wedge[(x \wedge y) \vee(x \wedge s)]=(t \wedge x \wedge y) \vee(t \wedge x \wedge s)
$$

\footnotetext{
* Corresponding author: shiuly.math.ru@yahoo.com
} 
The element $s$ is called neutral if

(i) $S$ is standard and

(ii) for all $x, y, z \in S, s \wedge[(x \wedge y) \vee(x \wedge z)]=(s \wedge x \wedge y) \vee(s \wedge x \wedge z)$.

In a distributive nearlattice every element is neutral and hence standard. An element $n$ in a nearlattice $S$ is called sesquimedial if for all $x, y, z \in S$, $\varangle(x \wedge n) \vee(y \wedge n)] \wedge[(y \wedge n) \vee(z \wedge n)] \vee(x \wedge y) \vee(y \wedge z)$ exists in $S$. An element $n$ of a nearlattice $S$ is called an upper element if $x \vee n$ exists for all $x \in S$. Every upper element is of course a sesquimedial element. An element $n$ is called a central element of $S$ if it is neutral, upper and complemented in each interval containing it.

For a fixed element $n$ of a nearlattice $S$, a convex subnearlattice of $S$ containing $n$ is called an $n$-ideal of $S$. For a medial element $n$ of a nearlattice $S$, an $n$-ideal $P$ of $S$ is called prime if $P \neq S$ and $m(x, n, y) \in P(x, y \in S)$ implies either $x \in P$ or $y \in P$.

A prime $n$-ideal $P$ is said to be a minimal prime $n$-ideal belonging to $n$-ideal $I$ if (i) $I \subseteq P$ and (ii) There exists no prime $n$ - ideal $Q$ such that $Q \neq P$ and $I \subseteq Q \subseteq P$. A prime $n$-ideal $P$ of a nearlattice $S$ is called a minimal prime $n$-ideal if there exists no prime $n$-ideal $Q$ such that $Q \neq P$ and $Q \subseteq P$.

Let $L$ be a lattice with 0 and $a \in L$. Then $a^{*}$ of $L$ is called a pseudocomplement of $a$ if $a \wedge a^{*}=0$ and if $a \wedge x=0$ for any $x \in L$ then $x \leq a^{*}$. A lattice $L$ is called pseudocomplemented if every element of $L$ has a pseudocomplement.

A nearlattice $S$ with $O$ is called sectionally pseudocomplemented if the interval $[0, x]$ for each $x \in S$, is pseudocomplemented. Of course, every finite distributive nearlattice is sectionally pseudocomplemented. A nearlattice $S$ is called relatively pseudocomplemented if the interval $[a, b]$ for each $a, b \in S, \quad a<b$ is pseudocomplemented.

A distributive nearlattice $S$ with $O$ is called a generalized Stone nearlattice if $(x]^{*} \vee(x]^{* * *}=S$ for each $x \in S$. A distributive nearlattice $S$ with 0 is a generalized Stone nearlattice if and only if each interval $[0, x], 0<x \in S$ is a Stone lattice.

For any n-ideal $\mathrm{J}$ of a nearlattice $\mathrm{S}$,

$$
J^{+}=\{x \in S: m(x, n, j)=n \text { for all } j \in J\}
$$

An $n$-ideal generated by a single element $a$ is called principal $n$-ideal, denoted by $\langle a\rangle_{n}$. The set of principal $n$-ideal is denoted by $P_{n}(S)$. When $S$ is a distributive nearlattice then for any $a \in S$ we define

$$
\begin{aligned}
\langle a\rangle_{n} & =\{y \in S: a \wedge n \leq y=(y \wedge a) \vee(y \wedge n)\} \\
& =\{y \in S: y=(y \wedge a) \vee(y \wedge n) \vee(a \wedge n)\}
\end{aligned}
$$

When $n$ is an upper element, then $\langle a\rangle_{n}$ is the closed interval $[a \wedge n, a \vee n]$.

We know that for a distributive nearlattice $S$ with an upper element $n, P_{n}(S)$ is a distributive nearlattice with the smallest element $\{n\}$. Let $\langle a\rangle_{n} \in P_{n}(S)$. By the interval $\left[\{n\},\langle a\rangle_{n}\right]$ in $P_{n}(S)$, we mean the set of all principal n-ideals contained in $\langle a\rangle_{n} \cdot P_{n}(S)$ is called sectionally pseudocomplemented if for each $\langle a\rangle_{n} \in P_{n}(S)$, the interval $\left[\{n\},\langle a\rangle_{n}\right]$ in $P_{n}(S)$ is pseudocomplemented. That is, each principal n-ideal contained 
in $\langle a\rangle_{n}$ has a relative pseudocomplement in $\left[\{n\},\langle a\rangle_{n}\right]$ which is also a member of $P_{n}(S)$. We shall denote the relative pseudocomplement of $\langle b\rangle_{n}$ in any interval by $\langle b\rangle_{n}^{0}$, while $\langle b\rangle_{n}^{+}$denotes the pseudocomplement of $\langle b\rangle_{n}$ in $I_{n}(S)$.

If $P_{n}(S)$ is a distributive sectionally pseudocomplemented nearlattice, then $P_{n}(S)$ is a generalized Stone nearlattice if for each $\langle a\rangle_{n} \in P_{n}(S)$, the interval $\left[\{n\},\langle a\rangle_{n}\right]$ in $P_{n}(S)$ is a Stone lattice.

For $b \leq a \leq n$, if $[b, n]$ is dual pseudocomplemented then $a^{0 d}$ denotes the relative dual pseudocomplement of $a$ in $[b, n]$. If $[n, d]$ is pseudocomplemented then for $c \in[n, d], c^{0}$ denotes the relative pseudocomplement of $c$ in $[n, d]$. Two prime n-ideals $P$ and $Q$ of a nearlattice $S$ are called comaximal if $P \vee Q=S$.

In this paper, we have given several characterizations of those $P_{n}(S)$ which are generalized Stone nearlattices in terms of n-ideals. we have also discussed on $O(P)$ and $n(P)$ and given some properties of $n(P)$. Moreover, when $P_{n}(S)$ is sectionally pseudocomplemented distributive nearlattice, then we have proved that $P_{n}(S)$ is generalized Stone if and only if each prime $n$-ideal contains a unique minimal prime $n$ ideal.

Following result is due to [7] which will be needed for the development of this paper.

Theorem 1.1. For an element $n$ of a nearlattice $S$, the following conditions are equivalent :

(i) $n$ is central in $S$

(ii) $\mathrm{n}$ is upper and the map $\Phi: P_{n}(S) \rightarrow(n]^{d} \times[n)$ defined by

$\Phi \ll a>_{n}=(a \wedge n, a \vee n)$ is an isomorphism, where $(n]^{d}$ represents the dual of the lattice $(n]$.

When $n$ is a central element of $S$ ( then of course, $n$ is upper, and so sesquimedial), then by Theorem 1.1, $P_{n}(S) \cong(n]^{d} \times[n)$. Thus we have the following result.

Theorem 1.2. Let $S$ be a nearlattice and $n \in S$ be a central element. Then $P_{n}(S)$ is sectionally pseudocomplemented if and only if $(n]$ is sectionally dual pseudocomplemented and $[n)$ is sectionally pseudocomplemented.

Corollary 1.3. Let $n$ be a central element and $P_{n}(S)$ be a sectionally pseudocomplemented distributive nearlattice. Then for $\{n\} \subseteq\langle a\rangle_{n} \subseteq\langle b\rangle_{n}$, $<a>_{n}^{0}=[a \wedge n, a \vee n]^{0}=\left[(a \wedge n)^{0 d},(a \vee n)^{0}\right]$.

Proof. Since $P_{n}(S)$ is sectionally pseudocomplemented, so by Theorem 1.2, $(n]$ is sectionally dual pseudocomplemented and $[n)$ is sectionally pseudocomplemented. Here $b \wedge n \leq a \wedge n \leq n \leq a \vee n \leq b \vee n$. 
Since $(a \wedge n)^{0 d}$ is the relative dual pseudocomplement of $a \wedge n$ in $[b \wedge n, n]$ and

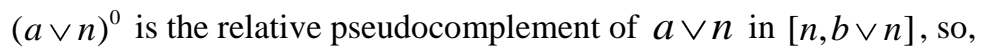

$[a \wedge n, a \vee n] \cap\left[(a \wedge n)^{0 d},(a \vee n)^{0}\right]=\left[(a \wedge n) \vee(a \wedge n)^{0 d},(a \vee n) \wedge(a \vee n)^{0}\right]$

$$
=[n, n]=\{n\} .
$$

Now let $t \in\left\langle a>_{n}^{0}\right.$. Then $[t \wedge n, t \vee n] \subseteq<a>_{n}^{0}$.

Thus, $\{n\}=[t \wedge n, t \vee n] \cap[a \wedge n, a \vee n]$

$$
=[(t \wedge n) \vee(a \wedge n),(t \vee n) \wedge(a \vee n)]
$$

and so $(t \wedge n) \vee(a \wedge n)=n=(t \vee n) \wedge(a \vee n)$.

This implies $(t \wedge n) \geq(a \wedge n)^{0 d}$ and $(t \vee n) \leq(a \vee n)^{0}$.

Hence, $[t \wedge n, t \vee n] \subseteq\left[(a \wedge n)^{0 d},(a \vee n)^{0}\right]$ and so $\left\langle a>_{n}^{0} \subseteq\left[(a \wedge n)^{0 d},(a \vee n)^{0}\right]\right.$.

Therefore $\langle a\rangle_{n}^{0}=\left[(a \wedge n)^{0 d},(a \vee n)^{0}\right]$.

If $S$ is a distributive lattice with 0 and 1 , then for a central element $n \in S$, $P_{n}(S)=F_{n}(S)$. Then $P_{n}(S)$ is pseudocomplemented if and only if $(n]$ is dual pseudocomplemented and $[n)$ is pseudocomplemented, as $F_{n}(S) \cong(n]^{d} \times[n)$. For any $n \leq b \leq 1, b^{+}$denotes the pseudocomplement of $b$ in $[n, 1]$, while for $0 \leq a \leq n, a^{+d}$ denotes the dual pseudocomplement of $a$ in $[0, n]$.

Corollary 1.4. Let $n$ be a central element of a lattice $S$ with 0,1 and $P_{n}(S)$ is a pseudocomplemented distributive lattice. Then for any $a \in S$,

$$
<a>_{n}^{+}=\left[(a \wedge n)^{+d},(a \vee n)^{+}\right] \text {. }
$$

A distributive nearlattice $S$ with 0 is generalized Stone nearlattice if for each $x \in S$, $(x]^{*} \vee(x]^{* *}=S$. By [6], a distributive nearlattice $S$ with 0 is a generalized Stone nearlattice if and only if each interval $[0, x], 0<x \in S$ is a Stone lattice.

To prove Theorem 1.7 we need the following lemmas. Lemma 1.5 is trivial by Theorem 1.2

Lemma 1.5. Suppose $n$ is a central element of a distributive nearlattice $S$, and $P_{n}(S)$ is sectionally pseudocomplemented. Then $P_{n}(S)$ is generalized Stone if and only if $(n]$ is dual generalized Stone and $[n)$ is generalized Stone.

Lemma 1.6. Suppose $P_{n}(S)$ is a sectionally pseudocomplemented distributive nearlattice. Let $x, y \in S$ with $\langle x\rangle_{n} \cap\langle y\rangle_{n}=\{n\}$. Then the following conditions are equivalent :

(i) $\langle x\rangle_{n}^{+} \vee\langle y\rangle_{n}^{+}=S$;

(ii) For any $t \in S, \quad\left\langle m(x, n, t)>_{n}^{0} \vee\left\langle m(y, n, t)>_{n}^{0}=\langle t\rangle_{n}\right.\right.$ where $\langle m(x, n, t)\rangle_{n}^{0}$ denotes the relative pseudocomplement of

$$
<m(x, n, t)>_{n} \text { in }\left[\{n\},<t>_{n}\right] \text {. }
$$


Proof. (i) $\Rightarrow$ (ii). Suppose (i) holds. Then for any $t \in S$,

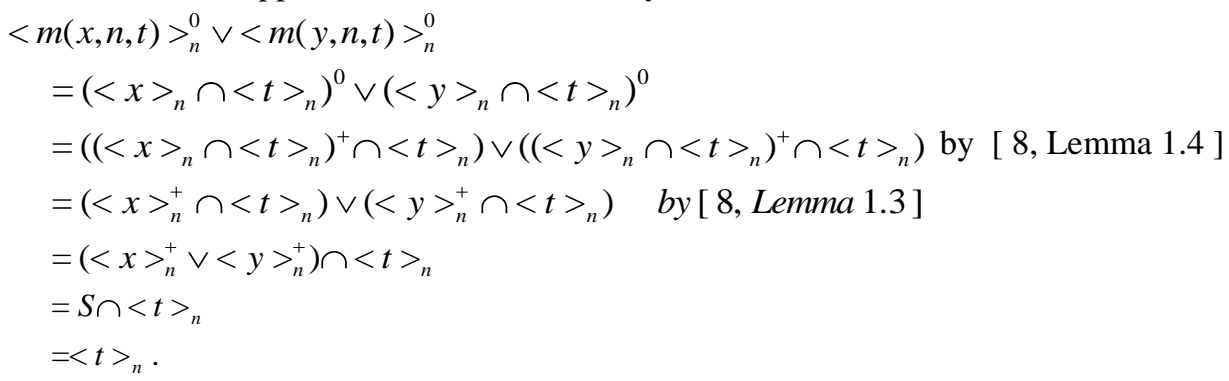

Hence (ii) holds.

(ii) $\Rightarrow$ (i). Suppose (ii) holds and $t \in S$.

By (ii), $\langle m(x, n, t)\rangle_{n}^{0} \vee\langle m(y, n, t)\rangle_{n}^{0}=\langle t\rangle_{n}$.

Then using [8, Lemmas 1.3 and 1.4] and the calculation of (i) $\Rightarrow$ (ii) above we get

$\left(\langle x\rangle_{n}^{+} \vee\langle y\rangle_{n}^{+}\right) \cap\langle t\rangle_{n}=\langle t\rangle_{n}$.

This implies $\langle t\rangle_{n} \subseteq\langle x\rangle_{n}^{+} \vee\langle y\rangle_{n}^{+}$and so $t \in\langle x\rangle_{n}^{+} \vee\langle y\rangle_{n}^{+}$.

Therefore, $\langle x\rangle_{n}^{+} \vee\langle y\rangle_{n}^{+}=S$.

Theorem 1.7. Let $n$ be a central element of $S$, and $P_{n}(S)$ be a sectionally pseudocomplemented distributive nearlattice. Then the following conditions are equivalent :

(i) $P_{n}(S)$ is generalized Stone ;

(ii) For any $x \in S, \quad\langle x\rangle_{n}^{+} \vee\langle x\rangle_{n}^{++}=S$;

(iii) For all $x, y \in S, \quad\left(\langle x\rangle_{n} \cap\langle y\rangle_{n}\right)^{+}=\langle x\rangle_{n}^{+} \vee\langle y\rangle_{n}^{+}$;

(iv) For all $x, y \in S, \quad\langle x\rangle_{n} \cap\langle y\rangle_{n}=\{n\}$ implies that $\langle x\rangle_{n}^{+} \vee\langle y\rangle_{n}^{+}=S$.

Proof. (i) $\Rightarrow$ (ii). Suppose (i) holds and, the $t \in S \mathrm{n}$ for any $x \in S$, $m(x, n, t) \in\langle t\rangle_{n}$ and so $\langle m(t, n, x)\rangle_{n} \in\left[\{n\},\langle t\rangle_{n}\right]$.

Since $P_{n}(S)$ is generalized Stone, so $\langle m(t, n, x)\rangle_{n}^{0} \vee\langle m(t, n, x)\rangle_{n}^{00}=\langle t\rangle_{n}$.

Then by [8, Lemma 1.4],

$$
\begin{aligned}
& \langle t\rangle_{n}=\left(\langle m(t, n, x)\rangle_{n}^{+} \cap\langle t\rangle_{n}\right) \vee\left(\langle m(t, n, x)\rangle_{n}^{++} \cap\langle t\rangle_{n}\right) \\
& =\left(\left(\langle x\rangle_{n} \cap\langle t\rangle_{n}\right)^{+} \cap\langle t\rangle_{n}\right) \vee\left(\left(\langle x\rangle_{n} \cap\langle t\rangle_{n}\right)^{++} \cap\langle t\rangle_{n}\right)
\end{aligned}
$$

Thus by [8, Lemma 1.3],

$$
\langle t\rangle_{n}=\left(\langle x\rangle_{n}^{+} \cap\langle t\rangle_{n}\right) \vee\left(\langle x\rangle_{n}^{++} \cap\langle t\rangle_{n}\right) .
$$

Thus $\langle t\rangle_{n}=\left(\langle x\rangle_{n}^{+} \vee\langle x\rangle_{n}^{++}\right) \cap\langle t\rangle_{n}$.

This implies $\langle t\rangle_{n} \subseteq\langle x\rangle_{n}^{+} \vee\langle x\rangle_{n}^{++}$and so $t \in\langle x\rangle_{n}^{+} \vee\langle x\rangle_{n}^{++}$.

Therefore $\langle x\rangle_{n}^{+} \vee\langle x\rangle_{n}^{++}=S$.

(ii) $\Rightarrow$ (iii). Suppose (ii) holds. 
For any $x, y \in S$

$$
\begin{aligned}
\left(\langle x\rangle_{n}\right. & \cap\left\langle y>_{n}\right) \cap\left(\langle x\rangle_{n}^{+} \vee\langle y\rangle_{n}^{+}\right) \\
= & \left(\langle x\rangle_{n} \cap\langle y\rangle_{n} \cap\langle x\rangle_{n}^{+}\right) \vee\left(\langle x\rangle_{n} \cap\langle y\rangle_{n} \cap\langle y\rangle_{n}^{+}\right) \\
= & \{n\} \vee\{n\}=\{n\}
\end{aligned}
$$

Now let $\langle x\rangle_{n} \cap\langle y\rangle_{n} \cap I=\{n\}$ for some n-ideal I.

Then $\langle y\rangle_{n} \cap I \subseteq\langle x\rangle_{n}^{+}$. Meeting $\langle x\rangle_{n}^{++}$with both sides,

we have $\langle y\rangle_{n} \cap I \cap\langle x\rangle_{n}^{++}=\{n\}$.

This implies $I \cap\langle x\rangle_{n}^{++} \subseteq\langle y\rangle_{n}^{+}$.

$$
\text { Hence } \begin{aligned}
I & =I \cap S \\
& =I \cap\left(\left\langlex>_{n}^{+} \vee\left\langle x>_{n}^{++}\right)\right.\right. \\
& =\left(I \cap\left\langle x>_{n}^{+}\right) \vee\left(I \cap\langle x\rangle_{n}^{++}\right)\right. \\
& \subseteq\langle x\rangle_{n}^{+} \vee\langle y\rangle_{n}^{+}
\end{aligned}
$$

Therefore, $\langle x\rangle_{n}^{+} \vee\langle y\rangle_{n}^{+}=\left(\langle x\rangle_{n} \cap\langle y\rangle_{n}\right)^{+}$.

(iii) $\Rightarrow$ (iv). Let $\langle x\rangle_{n} \cap\langle y\rangle_{n}=\{n\}$ for some $x, y \in S$.

Then by (iii),

$$
\begin{gathered}
S=\{n\}^{+}=\left(<x>_{n} \cap\left\langle y>_{n}\right)^{+}\right. \\
=<x>_{n}^{+} \vee<y>_{n}^{+} .
\end{gathered}
$$

Thus (iv) holds.

To complete the proof we shall show that (iv) $\Rightarrow$ (i).

Suppose (iv) holds. Since $P_{n}(S)$ is sectionally pseudocomplemented, so by Theorem 1.2, $(n]$ is sectionally dual pseudocomplemented and $[n$ ) is sectionally pseudocomplemented. Suppose $n \leq b \leq d$. Let $b^{0}$ be the relative pseudocomplement of $b$ in $[n, d]$.

Now $b^{0} \wedge b^{00}=n$.

Thus $\left\langle b^{0}\right\rangle_{n} \cap\left\langle b^{00}\right\rangle_{n}=\left[n, b^{0} \wedge b^{00}\right]=[n, n]=\{n\}$.

Also $\left\langle b^{0}\right\rangle_{n},\left\langle b^{00}\right\rangle_{n} \subseteq\langle d\rangle_{n}$. Then by equivalent conditions of (iv) given in Lemma 1.6, we have $\left\langle m\left(b^{0}, n, d\right)\right\rangle_{n}^{0} \vee\left\langle m\left(b^{00}, n, d\right)\right\rangle_{n}^{0}=\langle d\rangle_{n}$.

But $m\left(b^{0}, n, d\right)=b^{0}$ and $m\left(b^{00}, n, d\right)=b^{00}$ as $n \leq b^{0}, b^{00} \leq d$.

Since by Corollary 1.4, $\left\langle b^{0}\right\rangle_{n}^{0}=\left\langle b^{00}\right\rangle_{n}$ and $\left\langle b^{00}\right\rangle_{n}^{0}=\left\langle b^{000}\right\rangle_{n}=\left\langle b^{0}\right\rangle_{n}$.

Therefore, $\left.\langle d\rangle_{n}=\left\langle b^{00}\right\rangle_{n} \vee<b^{0}\right\rangle_{n}$

$$
=<b^{0} \vee b^{00}>_{n}
$$

which gives $b^{0} \vee b^{00}=d$. This implies $[n, d]$ is a Stone lattice.

That is, [n) is generalized Stone.

A dual proof of above shows that (iv) also implies that $(n]$ is a dual generalized Stone lattice. Therefore, by Lemma $1.5, P_{n}(S)$ is generalized Stone.

Following corollary is an immediate consequence of above result.

Corollary 1.8. Let $n$ be a central element of a distributive lattice $\mathrm{L}$ with 0 and 1 and 
let $P_{n}(L)$ be a pseudocomplemented distributive lattice. Then the following conditions are equivalent :

(i) $P_{n}(L)$ is Stone ;

(ii) For all $x \in L, \quad\langle x\rangle_{n}^{+} \vee\langle x\rangle_{n}^{++}=L$;

(iii) For all $x, y \in L, \quad\left(\langle x\rangle_{n} \cap\langle y\rangle_{n}\right)^{+}=\langle x\rangle_{n}^{+} \vee\langle y\rangle_{n}^{+}$;

(iv) For all $x, y \in L, \quad\langle x\rangle_{n} \cap\langle y\rangle_{n}=\{n\}$ implies that $\langle x\rangle_{n}^{+} \vee\langle y\rangle_{n}^{+}=L$.

For a prime ideal $P$ of a distributive nearlattice $S$ with 0 , we define $O(P)=\{x \in S: x \wedge y=0$ for some $y \in S-P\}$

Clearly $O(P)$ is an ideal and $0(P) \subseteq P$. Note that $0(P)$ is the intersection of all the minimal prime ideals of $\mathrm{S}$ which are contained in $P$.

For a prime n-ideal $P$ of a distributive nearlattice $S$, we write $n(P)=\{y \in S: m(y, n, x)=n$ for some $x \in S-P\}$.

Clearly, $n(P)$ is an n-ideal and $n(P) \subseteq P$.

Lemma 1.9. Let $S$ be a distributive nearlattice with a medial element $n$ and $P$ be a prime $\mathrm{n}$-ideal in $S$. Then each minimal prime $\mathrm{n}$ - ideal belonging to $n(P)$ is contained in $P$.

Proof. Let $Q$ be a minimal prime n-ideal belonging to $n(P)$. If $Q \nsubseteq P$, then choose $y \in Q-P$. Since $Q$ is a prime n-ideal, so by [9,Theorem 1.5], we know that $Q$ is either an ideal or a filter. Without loss of generality suppose $Q$ is an ideal. Now let

$T=\{t \in S: m(y, n, t) \in n(P)\}$.

We shall show that $T \nsubseteq Q$. If not, let $D=(S-Q) \vee[y)$.

Then $n(P) \cap D=\Phi$.

For otherwise, $y \wedge r \in n(P)$ for some $r \in S-Q$. Then by convexity,

$y \wedge r \leq m(y, n, r) \leq(y \wedge r) \vee n$ implies $m(y, n, r) \in n(P)$. Hence $r \in T \subseteq Q$, which is a contradiction.

Thus by [9,Theorem 1.9], there exists a prime n-ideal $R$ containing $n(P)$ disjoint to $D$. Then $R \subseteq Q$.

Moreover, $R \neq Q$ as $y \notin R$, this shows that $Q$ is not a minimal prime n-ideal belonging to $n(P)$, which is a contradiction.

Therefore $T \nsubseteq Q$. Hence there exists $z \notin Q$ such that $m(y, n, z) \in n(P)$. Thus $m(m(y, n, z), n, x)=n$ for some $x \in S-P$. It is easy to see that $m(m(y, n, z), n, x)=m(m(y, n, x), n, z)$.

Hence $m(m(y, n, x), n, z)=n$. Since $P$ is prime and $y, x \notin P$ so $m(y, n, x) \notin P$.

Therefore, $z \in n(P) \subseteq Q$, which is a contradiction.

Hence $Q \subseteq P$. 
Proposition 1.10. For a medial element $\mathrm{n}$ if $P$ is a prime $\mathrm{n}$ - ideal in a distributive nearlattice $S$, then $n(P)$ is the intersection of all minimal prime $\mathrm{n}$ - ideals contained in $P$.

Proof. Clearly $n(P)$ is contained in any prime n-ideal which is contained in $P$. Hence $n(P)$ is contained in the intersection of all minimal prime n-ideals contained in $P$.

Since $\mathrm{S}$ is distributive, so by [7, Corollary 2.1.10], $n(P)$ is the intersection of all minimal prime n-ideals belonging to it.

By [8, Lemma 1.2], as each prime n-ideal contains a minimal prime n-ideal, above remarks and Lemma 1.9 establish the proposition.

Following result has been proved by [5] for lattices. We generalize that result for nearlattices with the help of [10,Theorem 1.7].

Theorem 1.11. Let $P_{n}(S)$ be a sectionally pseudocomplemented distributive nearlattice and $n$ be central element in $\mathrm{S}$. Then the following conditions are equivalent :

(i) For any $x \in S, \quad\langle x\rangle_{n}^{+} \vee\langle x\rangle_{n}^{++}=S$, equivalently, $P_{n}(S)$ is generalized Stone;

(ii) For any two minimal prime n-ideals $P$ and $Q, P \vee Q=S$;

(iii) Every prime $\mathrm{n}$ - ideal contains a unique minimal prime $\mathrm{n}-\mathrm{ideal}$;

(iv) For each prime n- ideal $P, n(P)$ is a prime n- ideal.

Proof. (i) $\Rightarrow$ (ii). Suppose (i) holds.

Let $x \in P-Q$. Then $\langle x\rangle_{n} \subseteq P-Q$. Now, $\langle x\rangle_{n} \cap\langle x\rangle_{n}^{+}=\{n\} \subseteq Q$.

So $\langle x\rangle_{n}^{+} \subseteq Q$ as $Q$ is prime.

Again, $x \in P$ implies $\langle x\rangle_{n}^{++} \subseteq P$ by [8, Theorem 1.6].

Hence by (i), $S=\langle x\rangle_{n}^{+} \vee\langle x\rangle_{n}^{++} \subseteq Q \vee P$. Therefore, $P \vee Q=S$.

(ii) $\Leftrightarrow$ (iii) is trivial.

(iii) $\Rightarrow$ (iv) is direct consequence of Proposition 1.10 .

(iv) $\Rightarrow$ (i). Suppose (iv) holds.

First we shall show that for all $x, y \in S$ with $\langle x\rangle_{n} \cap\langle y\rangle_{n}=\{n\} \quad$ implies $\langle x\rangle_{n}^{+} \vee\langle y\rangle_{n}^{+}=S$. If it does not hold, then there exist

$x, y \in S$ with $\langle x\rangle_{n} \cap\langle y\rangle_{n}=\{n\}$ such that $\langle x\rangle_{n}^{+} \vee\langle y\rangle_{n}^{+} \neq S$.

As $S$ is distributive, so by [9, Theorem 1.9], there is a prime n-ideal $P$ such that $\langle x\rangle_{n}^{+} \vee\langle y\rangle_{n}^{+} \subseteq P$. Then $\langle x\rangle_{n}^{+} \subseteq P$ and $\langle y\rangle_{n}^{+} \subseteq P$ imply $x \notin n(P)$ and $y \notin n(P)$.

By (iv), $n(P)$ is prime n-ideal and so $m(x, n, y)=n \in n(P)$ is contradictory.

Thus for all $x, y \in S$ with $\langle x\rangle_{n} \cap\langle y\rangle_{n}=\{n\}$

implies that $\langle x\rangle_{n}^{+} \vee\langle y\rangle_{n}^{+}=S$.

Hence by equivalent conditions of Theorem 1.7, (i) holds. 


\section{References}

1. R. Balbes and A. Horn, Duke Math. J. 38, 537 (1971). http://dx.doi.org/10.1215/S0012-7094-71-03843-9

2. W. H. Cornish, Austral. Math. Soc. 14, 200 (1972). http://dx.doi.org/10.1017/S1446788700010041

3. T. Katrinak, (Russian) Math. Fyz. Casopis 16, 128 (1966).

4. T. Katrinak, (Russian) Math. Casopis Sloven. Akad. Vied. 17, 20 (1967).

5. A. S. A. Noor and M. A. Ali, The Rajshahi University Studies, Part-B 26, 83(1998).

6. A. S. A. Noor and A. K. M. S. Islam, J. Sci. Jahangirnagar University 33 (1), 105 (2010).

7. S. Akhter, A Study of Principal n-Ideals of a Nearlattice, Ph.D. Thesis, Rajshahi University, Rajshahi (2003).

8. S. Akhter and M. A. Latif, J. Sci. 35, 217 (2007).

9. S. Akhter and A. S. A. Noor, Ganit J. Bangladesh Math. Soc. 24, 35 (2005).

10. S. Akhter and A. S. A. Noor, J. Sci. Res. 4 (3), 589 (2012). http://dx.doi.org/10.3329/jsr.v4i3.10103 